\title{
Mitochondrial disruption in peroxisome deficient cells is hepatocyte selective but is not mediated by common hepatic peroxisomal metabolites
}

Abhijit Babaji Shinde ${ }^{a}$, Ritesh Kumar Baboota ${ }^{a}$, Simone Denis ${ }^{b}$, Ursula Loizides-Mangold ${ }^{c 1}$, Annelies Peeters $^{a}$, Marc Espeel ${ }^{d}$, Ana Rita Malheiro ${ }^{e}$, Howard Riezman ${ }^{c}$, Stefan Vinckier ${ }^{\ddagger}$, Frédéric $M V^{2} z^{b}$, Pedro Brites $^{\mathrm{e}}$, Sacha Ferdinandusse ${ }^{\mathrm{b}}$, Paul P Van Veldhoveng2, Myriam Baes ${ }^{\mathrm{a} 2}$

${ }^{a} K U$ Leuven - University of Leuven, Department of Pharmaceutical and Pharmacological Sciences, Laboratory of Cell Metabolism, B-3000 Leuven, Belgium

'Laboratory Genetic Metabolic Diseases, Department of Clinical Chemistry, Academic Medical Center, University of Amsterdam, Amsterdam, Netherlands

'Department of Biochemistry, NCCR Chemical Biology, University of Geneva, 1211 Geneva, Switzerland

${ }^{d}$ Dept Basic Medical Sciences, UGhent, B-9000 Ghent, Belgium

e Neurolipid Biology group, Instituto de Biologia Molecular e Celular - IBMC and Instituto de Inovação e Investigação em Saúde, University of Porto, 4200-135 Porto, Portugal

${ }^{f}$ VIB-KU Leuven Centre for Cancer Biology, Laboratory of Angiogenesis and Vascular Metabolism, B3000 Leuven, Belgium

gKU Leuven - University of Leuven, Department of Cellular and Molecular Medicine, Laboratory for Lipid Biochemistry and Protein Interactions, KU Leuven, B-3000 Leuven, Belgium

${ }^{1}$ current affiliation: Division of Endocrinology, Diabetes and Nutrition, Department of Clinical Medicine, Faculty of Medicine, University of Geneva, Switzerland

${ }^{2}$ equal last authors

Corresponding authors:

Myriam Baes, PhD

Laboratory for Cell Metabolism

Faculty of Pharmaceutical and Pharmacological Sciences

Campus Gasthuisberg O/N2

Herestraat 49

B 3000 Leuven

Tel + 3216330853

e-mail: Myriam.Baes@kuleuven.be

Paul P. Van Veldhoven, PhD

LIPIT

Department of Cellular and Molecular Medicine

Campus Gasthuisberg O/N1

Herestraat 49

B 3000 Leuven

Tel + 3216330686

e-mail: Paul.Vanveldhoven@kuleuven.be 


\section{Abstract}

The structural disruption of the mitochondrial inner membrane in hepatocytes lacking functional peroxisomes along with selective impairment of respiratory complexes and depletion of mitochondrial DNA was previously reported. In search for the molecular origin of these mitochondrial alterations, we here show that these are tissue selective as they do neither occur in peroxisome deficient brain nor in peroxisome deficient striated muscle. Given the hepatocyte selectivity, we investigated the potential involvement of metabolites that are primarily handled by hepatic peroxisomes. Levels of these metabolites were manipulated in L-Pex5 knockout mice and/or compared with levels in different mouse models with a peroxisomal $\beta$-oxidation deficiency. We show that neither the deficiency of docosahexaenoic acid nor the accumulation of branched chain fatty acids, dicarboxylic acids or C27 bile acid intermediates are solely responsible for the mitochondrial anomalies. In conclusion, we demonstrate that peroxisomal inactivity differentially impacts mitochondria depending on the cell type but the cause of the mitochondrial destruction needs to be further explored.

\section{Highlights}

- Severe mitochondrial destruction in peroxisome deficient cells is hepatocyte selective

- Isoprenoid-derived branched chain or dicarboxylic fatty acid overload alone does not induce the mitochondrial deficits

- Shortage of docosahexaenoic acid and accumulation of bile acid intermediates do not correlate with pathology

\section{Keywords}

mitochondria, peroxisomes, respiratory chain, Zellweger, bile acids, $\beta$-oxidation

\section{Abbreviations}

ACOX, acyl-CoA oxidase; DCA, dicarboxylic fatty acid, DHA, docosahexaenoic acid; DHCA, 3a,7adihydroxycholestanoic acid; MCK, muscle creatine kinase; MFP, multifunctional protein; PBD, peroxisome biogenesis disorder; PUFA, polyunsaturated fatty acid; ROS, reactive oxygen species;

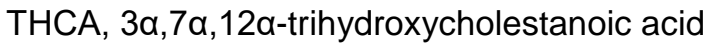




\section{Introduction}

In some early reports on patients with the most severe form of a peroxisome biogenesis disorder (PBD), Zellweger syndrome, mitochondrial aberrations were documented. In hepatocytes mitochondria with severely distorted cristae and altered appearance of the matrix were found [1-3]. However, the anomalies were variable in severity and in a few other patients no abnormalities were detected [4]. Milder mitochondrial ultrastructural changes were sporadically reported in brain and in muscle [1, 4-7]. With regard to functional deficits, data from patients are even scarcer, and disparate impairments of complexes I - IV were reported in liver, muscle and brain [1, 8].

Mitochondrial anomalies were recapitulated in mice lacking PEX5 or PEX2, models for Zellweger syndrome $[9,10]$, confirming that primary peroxisomal defects cause secondary mitochondrial dysfunction in hepatocytes. In global Pex5 $\%$ and Pex2 ${ }^{-}$mice that die neonatally, the majority of hepatic mitochondria were severely affected with unusually shaped and sparse cristae $[10,11]$. Spectrophotometric analyses revealed that activities of the respiratory complexes I and $\mathrm{V}$ were markedly reduced. In other tissues, such as heart, adrenals, smooth muscle cells and kidney, mild structural changes were noticed such as electron dense inclusions and enlargement of the intermembrane space but cristae were not affected to the same extent as in hepatocytes [11].

More systematic studies on the links between loss of functional peroxisomes and mitochondrial integrity

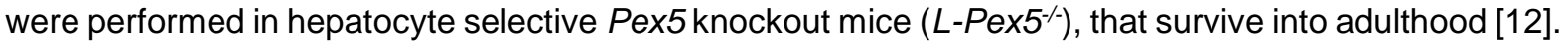
The severe distortion of cristae was accompanied with multiple metabolic and functional changes. The activities of complex I (<30\% of control), and complex III and V ( 60\% of control) were reduced, whereas the activities of complex II and IV were unaltered. This resulted in impaired oxygen consumption and ATP production and compensatory metabolic changes driven by AMPK activation [13]. A remarkable finding was that complex $V$ primarily occurred as sub-complexes that were not integrated in the membrane [14]. Furthermore, the expression of mitochondrial-encoded subunits was reduced concomitant with a decrease of the mitochondrial DNA/nuclear DNA ratio whereas nuclear encoded subunits were normal or even elevated [14].

The causal relationship between peroxisome inactivity and the mitochondrial perturbations in hepatocytes remains to date unresolved. Here, we investigated the molecular links by first addressing 
the tissue selectivity of the peroxisome-mitochondrial interaction. For this, the mitochondrial compartment was investigated in mice with selective inactivation of PEX5 in striated muscle and brain, tissues highly dependent on intact mitochondrial function [15].

As we found that mitochondrial ultrastructure and complex I activity were preserved in peroxisome deficient brain, heart and skeletal muscle, we subsequently hypothesized that metabolites that are in particular deregulated in peroxisome deficient hepatocytes and less in other cell types could be the triggering factor. Several metabolic conversions are indeed preferentially handled by hepatic peroxisomes including the synthesis of docosahexaenoic acid (DHA) and the degradation of branched chain fatty acids (i.e. phytanic and pristanic acid), dicarboxylic acids (DCAs) and the shortening of the side chain of the C27-bile acid intermediates $3 \alpha, 7 \alpha$-dihydroxycholestanoic acid (DHCA) and $3 \alpha, 7 \alpha, 12 \alpha-$ trihydroxycholestanoic acid (THCA) [16]. Notably, each of these metabolites was shown before to affect the respiratory chain. We investigated whether any of these metabolites could be the causative factors by measuring and manipulating their levels and relating these to mitochondrial function. Because the suspected metabolites are all dependent on peroxisomal $\beta$-oxidation, we performed parallel analyses in mice lacking enzymes of this pathway, either multifunctional protein 1 (MFP1 also called L-BP) [17] or multifunctional protein 2 (MFP2 also called D-BP/HSD17B4) [18]. 


\section{Materials and methods}

\subsection{Mouse breeding and diets}

Global Pex5 knockout mice were previously described [11]. Tissue selective Pex5 knockout mice were

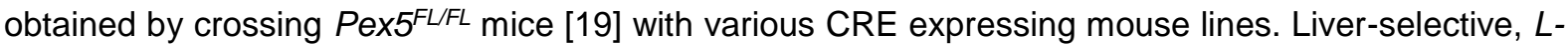
$P e x 5 \%$ mice [12] and neural cell-selective Nestin-Pex5 $\%$ mice [20] were already reported. Pex5 ${ }^{F L F L}$ were used as controls. Selective knockout of Pex5 in striated muscle was obtained by using Mck-Cre mice

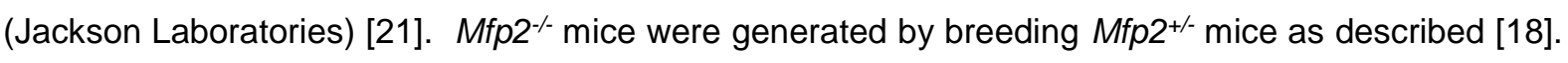
All mice were in Swiss/Webster background with the exception of the Mfp1 knockout mouse line that was in a mixed 129/Ola-C57BI/6 background [17]. DHA (10 mg/mouse every two days) protected from oxidation through gelatin microencapsulation (MEG-3, Ocean Nutrition Inc.) was supplemented by gavage [22] to 4 weeks old L-Pex5 ${ }^{-/}$mice and control mice for a period of 4 weeks. Some other adult wild type mice were fed a phytol diet (0.5\% in chow) for 2 weeks [23]. Mice were bred in the specific pathogen free animal housing facility of the KU Leuven, had ad libitum access to water and standard rodent food, and were kept on a 12-hour light and dark cycle. All animal experiments were performed in accordance with the "Guidelines for Care and Use of Experimental Animals" and fully approved by the Research Ethical committee of the KU Leuven (\#190/2012). Liver tissue from Mfp 1\% and control mice fed a coconut diet was collected previously [24].

\subsection{Metabolic analyses}

Livers were collected, snap frozen and kept at $-80^{\circ} \mathrm{C}$ until further use. The following metabolites were analyzed according to previously established procedures: bile acids by HPLC-negative ion electrospray tandem mass spectrometry [25], pristanic and phytanic acid by GC-MS [26, 27], DCAs by HPLC-MS [24] and plasmalogens by HPLC [20]. To quantify DHA in mitochondria, a crude mitochondrial fraction was isolated by differential centrifugation and further subjected to Percoll gradient centrifugation as described [14]. Lipids were transmethylated in $1 \mathrm{ml} 3 \mathrm{M} \mathrm{HCl}$ by incubating for 4 hours at $90^{\circ} \mathrm{C}$ in the presence of $10 \mathrm{nmol}$ internal standard; the methyl ester of 18-methylnonadecanoic acid. After cooling, the aqueous layer was extracted in $2 \mathrm{ml}$ hexane, and this extract was taken to dryness under nitrogen flow and resuspended in $80 \mu \mathrm{l}$ of hexane. One microliter of this solution was injected into a Hewlett Packard GC 5890 equipped with an Agilent J\&W HP-FFAP, 25m, 0.20mm, $0.33 \mu \mathrm{m}$ GC Column and 
eluting fatty acid methylesters were detected by flame ionization detection. Fatty acid concentrations were calculated using the known amount of internal standard and expressed as nmol per phosphorus content of the phospholipid fraction [28].

\subsection{Measurement of complex I activity}

Complex I activity was determined as described previously [11] with minor modifications. Briefly, liver (from 2-weeks old and 4-8 weeks old mice), corpus callosum, spinal cord and brain cortex (from 12weeks old mice), and heart (from 15-weeks old mice) were homogenized in 19 volumes of homogenization buffer (210 mM mannitol, 70 mM sucrose, 5 mM HEPES, 1 mM EGTA, pH 7.2) using a borosilicate glass hand homogenizer on ice. Homogenates were subjected to a sonication step (4 cycles of 10 seconds at amplitude 4, separated by a 20 second break) with a Soniprep 150 sonicator (MSE Scientific Instruments, Sussex, England). The decrease in NADH absorbance after oxidation by complex I in the presence of coenzyme Q was followed on a Perkin Elmer Lambda 25 UV/VIS spectrophotometer for 5 minutes. The reaction was carried out at $30^{\circ} \mathrm{C}$. Results were expressed as $\mathrm{U} / \mathrm{mg}$ of protein.

\subsection{Electron microscopy}

Mice were perfusion-fixed with $4 \%$ paraformaldehyde/0.05\% glutaraldehyde $(\mathrm{v} / \mathrm{v})$ for electron microscopy [29]. Isolated brains were post-fixed by immersion in $4 \%$ glutaraldehyde in $0.1 \mathrm{M}$ sodium cacodylate buffer ( $\mathrm{pH} 7.4)$ at room temperature for 1 day and then at $4{ }^{\circ} \mathrm{C}$ for 2 days. Brain samples were processed for ultrastructural analysis as described [30].

\subsection{Statistical analyses}

Statistical analysis was done using GraphPad Prism 5.01 software (CA, USA). Statistical significance was determined by performing either unpaired t-test; one-way ANOVA or two-way ANOVA based on the experimental design followed by Bonferroni test as required. 


\section{Results}

\subsection{Tissue selectivity of mitochondrial abnormalities in peroxisome deficiency}

In order to investigate the tissue selectivity of the severe anomalies at the inner mitochondrial membrane seen in adult PEX5 deficient hepatocytes, we checked mitochondrial structure and function in some other tissue selective Pex5 knockouts. As a readout, complex I activity, that is the most severely affected respiratory chain complex in hepatocytes, was monitored [12]. First, we checked whether, following albumin-Cre induced recombination of the Pex5 gene in hepatocytes, destruction of the inner mitochondrial membrane already occurred at an early time point. At the age of 2 weeks, the majority of mitochondria contained a paucity of cristae that were severely distorted. This was accompanied by reduced complex I activity in liver, similar to what was observed in adult liver (Fig. 1a,b). Subsequently, we inspected mitochondria in Nestin-Pex5 ${ }^{-}$mice that lack functional peroxisomes in neural cells and that develop severe neurodegeneration with inflammatory demyelination and neuron loss [20,31]. Remarkably, in contrast to the severe reduction of complex I activity in livers of $L-P e x 5^{-1-}$ mice, this complex was normally active in corpus callosum (Fig. 1c), neocortex and spinal cord (Suppl Fig1a,b) of Nestin-Pex5\% mice. Also by ultrastructural analysis, the mitochondrial anomalies seen in peroxisome deficient hepatocytes were not found in corpus callosum and cortex of Nestin-Pex5 ${ }^{-/}$mice despite severe deterioration of the tissue (Fig. 1d and suppl Fig. 1c). We subsequently examined mitochondria in mice with Pex5 inactivation in striated muscle obtained by breeding Pex5 FL/FL mice with Mck-Cre mice. Selective recombination of the Pex5 gene in the targeted tissue was confirmed by the absence of the full length transcript in heart and skeletal muscle but not in liver, kidney or spleen (Suppl Fig. 2a). Furthermore, the inactivation of peroxisomal function was illustrated by strongly reduced levels of plasmalogens in heart and skeletal muscle (Suppl Fig. 2b,c). Mck-Pex5 mice did not develop an overt phenotype under basal conditions and had a normal life span (to be published elsewhere). Spectrophotometric analysis revealed that in heart tissue of Mck-Pex $5^{-/}$mice complex I activity was unaffected (Fig. 1e). The preservation of mitochondria in muscle was further confirmed by electron microscopy showing normal morphology of mitochondria in heart (Fig. 1f). These results suggest that mitochondria are differentially affected according to the cell type in which peroxisome biogenesis is defective. This is further supported by our previous observation that complex I activity was normal in 
mouse embryonic $P$ ex5 ${ }^{-/}$fibroblasts [11]. This raised the question whether a reduction or accumulation of metabolites that are preferentially generated or degraded by hepatic peroxisomes, respectively, and less by other tissues are causative for the mitochondrial defects. We therefore explored whether deficiency of DHA, or accumulation of branched chain fatty acids, immature C27 bile acids or DCAs could underlie the mitochondrial defects.

\subsection{Restoration of depleted DHA does not improve mitochondrial anomalies}

The synthesis of the polyunsaturated fatty acid (PUFA) DHA depends on peroxisomal $\beta$-oxidation and occurs primarily in hepatocytes [32, 33]. We previously showed that DHA was markedly reduced in mitochondria isolated from $L-P e x 5^{-/}$mice as well as in different phospholipid fractions of these mitochondria [14]. In order to restore the levels of this PUFA, we treated the mice with DHA orally (10 $\mathrm{mg} / \mathrm{mouse}$ every two days starting at the age of 4 weeks). After the treatment, DHA was replenished in total hepatic lipids (not shown) as well as in mitochondria of $L-P e \times 5^{-/}$mice, increasing slightly above the levels in mitochondria of control mice with or without DHA supplementation (Fig. 2a). However, the activity of complex I did not improve, remaining below $20 \%$ of control values (Fig. $2 \mathrm{~b}$ ). Taken together these results indicate that the deficiency of DHA is not the source of the mitochondrial abnormalities.

\subsection{No evidence for the involvement of branched chain fatty acids in mitochondrial aberrations in L-Pex5 ${ }^{-/}$mice}

The catabolism of the branched chain fatty acid phytanic acid requires the consecutive action of peroxisomal $\alpha$ - and $\beta$-oxidation generating pristanic acid as an intermediate. Phytanic acid is derived from the dietary precursor phytol. Mice fed normal chow are only exposed to low levels of phytol and branched chain fatty acids. Still, we have previously reported increased concentrations of both phytanic and pristanic acids in the livers of $L-P e x 5^{-/}$mice amounting to 0.16 and $0.69 \mathrm{pmol} / \mathrm{nmol}$ phospholipids, respectively, which was about 20 fold higher than in wild type mice [12]. According to several in vitro experiments these branched chain fatty acids are toxic for mitochondria [34]. In order to investigate whether the complex I impairments in L-Pex5 knockout mice may be caused by accumulating branched chain fatty acids, we treated wild type mice with the precursor phytol ( $0.5 \%$ in chow for 2 weeks). This induced an increase of phytanic acid levels in liver to 8 (male) and 61 (female) pmol/nmol and of pristanic acid to 26 (male) and 48 (female) pmol/nmol phospholipid [23]. Remarkably, although these 
levels were several fold higher than in liver of $L-P e x 5$ knockout mice on normal chow, complex I activity was not impaired in the liver of these treated mice (Fig. 3).

\subsection{DCAs do not accumulate in PEX5 deficient liver}

DCAs are formed in the endoplasmic reticulum (ER) through $\omega$-hydroxylation of fatty acids by members of the Cyp4a family of cytochrome P450 enzymes that are enriched in liver and kidney [35]. These enzymes are positively regulated by PPARa [36] and we previously demonstrated that they are markedly upregulated in the livers of $L-P e \times 5^{-/}$mice $[12,37]$. The breakdown of long and medium chain DCAs occurs through peroxisomal $\beta$-oxidation. Hence, the increased formation and hampered degradation of DCA in hepatocytes lacking functional peroxisomes is expected to lead to the accumulation of DCAs which could potentially be toxic for mitochondria [38-40]. We therefore determined the concentrations of DCAs of different carbon lengths in the livers of $L-P e x 5^{-/}$and littermate control mice. Surprisingly, we did not find increased levels of short or medium length DCAs in the livers of $\mathrm{L}-\mathrm{Pex5^{-/ }}$ mice, and long chain DCAs were not detectable (Fig. 4a). To further investigate the possibility that DCAs cause mitochondrial anomalies, we measured complex I activity in a mouse model in which it was proven that DCAs accumulate. Indeed, Mfp $1^{1 /-}$ mice fed with coconut diet, a rich source of C12 fatty acids, were recently reported to accumulate C4 - C14 DCAs [24]. Analyses of complex I activity in liver specimens of the mice previously used for DCA quantification, revealed normal function when compared to littermate controls (Fig. 4b). Together, these observations do not support the possibility that an accumulation of DCA is responsible for causing the mitochondrial abnormalities in the livers of $L-P e \times 5 \%$ mice.

\subsection{Accumulating immature C27 bile acids do not underlie mitochondrial abnormalities in PEX5 deficient liver}

Hepatic peroxisomes are pivotal for the conversion of cholesterol into mature bile salts, as both the side chain shortening and the conjugation steps occur in this organelle, and bile acid synthesis primarily takes place in the liver. As expected, in liver of $L-P e x 5^{-/}$mice immature $\mathrm{C} 27$ bile acids accumulated and mature C24 bile acids were severely reduced, resulting in a marked increase of the C27/C24 bile acid ratio (sum of conjugated and unconjugated species) when compared to control littermates (Fig. 5a and Table 1). Whereas the bulk of C24 bile acids were taurine conjugated in both genotypes, the majority of $\mathrm{C} 27$ bile acids in L-Pex5 $\%$ liver occurred in the unconjugated form (Table 1). It was thereby 
striking that levels of DHCA were 3-fold higher than the more hydrophilic THCA (Table 1) that is formed by 12-hydroxylation of 4-cholesten-7a-ol-3-one during the synthesis of bile acids. Importantly, Ferdinandusse et al previously showed that unconjugated DHCA was the most toxic of a panel of bile acids, impairing cell viability and ATP production in isolated mitochondria [41].

To examine whether the altered bile acid content could cause the mitochondrial anomalies, we compared the bile acid profiles and mitochondrial integrity in livers of $L-P e x 5^{-}$and $M f p 2^{-/}$mice, another mouse model with impaired bile acid synthesis. MFP2 is necessary for the side chain shortening of immature C27 bile acids as it catalyzes two steps in the peroxisomal $\beta$-oxidation. The inactivity of MFP2 caused the predicted increase of the C27/C24 ratio (sum of conjugated and unconjugated species),

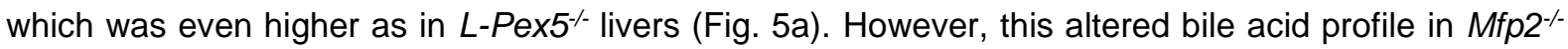

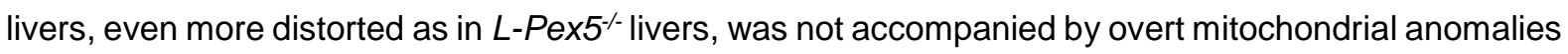
as complex I activity was unaltered in Mfp2--/ivers (Fig. 5b), which was in line with previous observations of conserved mitochondrial ultrastructure [42].

Closer inspection of the accumulating C27 bile acids, revealed that besides a similar accumulation of unconjugated $\mathrm{C} 27$ bile acids in both models, conjugated $\mathrm{C} 27$ bile acids were markedly more elevated

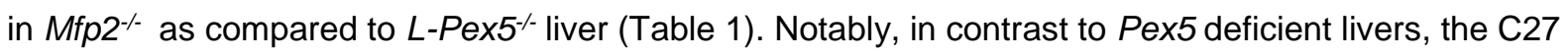
bile acid species in $\mathrm{Mfp}^{-}$- livers comprised more THCA than DHCA (Table 1) and both were primarily present in their 24-enoic form, consistent with intact peroxisomal acyl-CoA oxidase 2 (ACOX2) activity [16].

It thus appeared that the level of the presumably most toxic bile acid, unconjugated DHCA, was 5-fold

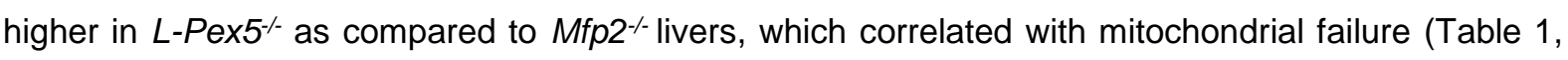
Figure 5d). To investigate whether the high DHCA levels are the cause of the mitochondrial disruption

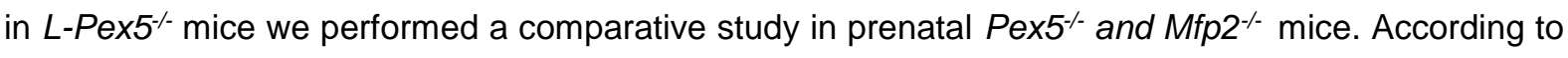
recent information, levels of bile acids and their synthesizing enzymes are low prenatally and are strongly induced on the day of birth in mice [43]. So, we turned to constitutive Pex5 knockout mice, in which we previously observed mitochondrial anomalies already one day before birth [11] and checked whether DHCA and THCA levels were already affected at this age. As a comparison we also measured the levels of these bile acid intermediates at the same age in Mfp2- liver in which no mitochondrial abnormalities were detected. Importantly, we found that in E18.5 livers unconjugated THCA levels were 
already grossly elevated in both $\mathrm{Mfp}^{2-}$ and $\mathrm{Pex}^{-}$livers reaching levels similar to those in adult mice (Fig. 5c and Table 1). In contrast, DHCA levels were much lower in E18.5 compared to adult livers and again comparable in both genotypes (Fig. 5d). Thus, the accumulation of DHCA (L-Pex $5^{-/}$adult $>>>M f p 2$

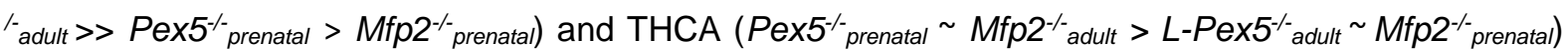
do not correlate with the structural and functional mitochondrial anomalies that were observed in both prenatal and adult Pex5 knockouts but not in Mfp2-deficient liver. Therefore, the abnormal bile acid profile does not appear to be the cause of the mitochondrial failure in Pex5/- livers. 


\section{Discussion}

Peroxisomes and mitochondria have been shown to have close interplay with respect to various biochemical and cell biological aspects; most important being the co-operation during the fatty acid oxidation process and detoxification of oxygen radicals. In our previous work we have shown that in hepatocytes mitochondrial integrity depends on intact peroxisomal function. In the present study we investigated whether the mitochondrial abnormalities are caused by an accumulation or shortage of peroxisomal metabolites. We showed that the impaired mitochondrial function is not due to reduced levels of DHA or to accumulation of the branched chain fatty acids phytanic and pristanic acid, dicarboxylic fatty acids, or the peroxisomal C27 bile acid intermediates.

The suspicion that peroxisomal metabolites could be causative factors was raised by the finding that the mitochondrial impairment due to peroxisomal dysfunction is cell type selective. An important observation in this respect has been that the structure and function of the mitochondrial inner membrane was preserved in the central nervous system and in striated muscle that are selectively depleted from intact peroxisomes. This differs from the mild and sporadic changes in mitochondrial structure in muscle tissue of global Pex5 knockouts [11] and we therefore assume that the latter were induced by non-cell autonomous mechanisms. In addition to brain and muscle, we previously showed that activities of the electron transport chain complexes were normal in Pex5 deficient fibroblasts [11]. Together, our data do not prove absolute cell type selectivity, but they suggest that a metabolic alteration that mainly takes place in hepatocytes is responsible for the mitochondrial damage. It can however not be excluded that the differential mitochondrial impairment is merely related to the larger volume of the peroxisomal compartment in hepatocytes. It is indeed well known that peroxisomes in hepatocytes are more numerous and larger in size compared to other tissues where they are often named microperoxisomes. It should be noted that mitochondrial dysfunction was reported in other situations of a primary peroxisomal defect e.g. in B12 oligodendrocytes and U87 astrocytes from which the ATP binding cassette subfamily $\mathrm{D}$ member $1(A B C D 1)$ was deleted [44]; in fibroblasts from X-ALD patients and in spinal cords of $A b c d 1 \%$ mice [45], and in muscle biopsies from patients with a mutation in PEX12 or PEX16 [6]. However, the mitochondrial distress in these cell types was much less pronounced than in 
L-Pex5 $\%$ liver with regard to respiratory complex activities and structure of the inner mitochondrial membrane.

For some metabolic processes, peroxisomes in hepatocytes seem to be less essential compared to other tissues. We previously found normal levels of plasmalogens and very long chain fatty acids in liver of $L-P e \times 5^{-/}$mice [12] which is in contrast with their respective depletion and accumulation in brain of Nestin-Pex5 $5^{-/}$mice [20]. This allowed to disregard these peroxisomal metabolites as the source of the mitochondrial anomalies in hepatocytes.

The synthesis of DHA, which primarily takes place in liver [46], requires one cycle of peroxisomal $\beta$ oxidation, explaining the shortage of this PUFA in livers without functional peroxisomes. We previously showed that DHA levels were reduced in all phospholipid classes in isolated mitochondria from Pex5 knockout livers, including in cardiolipin [14]. Alterations in the PUFA composition of mitochondrial phospholipids has diverse consequences on mitochondrial function such as impairments of mitochondrial permeability transition pore causing mitochondrial depolarization, uncoupling, swelling, defects of oxidative phosphorylation, loss of ATP production and cytochrome c release leading to apoptosis or necrosis [47-49]. We could however exclude that the reduced levels of DHA are the cause of the mitochondrial anomalies in PEX5 deficient hepatocytes as replenishing the liver with exogenous DHA did not improve mitochondrial function. An additional argument pleading against a role of DHA is that the pathological peroxisomal-mitochondrial connection is cell autonomous [12]. A minority of hepatocytes that escape loss of PEX5 and maintain import competent peroxisomes also harbor intact mitochondria, sharply delineated from neighboring cells with destroyed peroxisomal and mitochondrial compartments. In contrast, DHA produced by intact hepatocytes is expected to distribute in the liver and to other tissues of the organism.

The branched chain fatty acids phytanic and pristanic acid were shown to impair mitochondria in various ways including the membrane potential, $\mathrm{NAD}(\mathrm{P}) \mathrm{H}$ levels, activity of respiratory complexes, production of reactive oxygen species (ROS), opening of the mitochondrial transition pore, size and shape [50-52]. These fatty acids derive from phytol liberated from chlorophyll in ruminants and are taken up by humans when ingesting dairy products and meat. Failure of peroxisomal $\alpha$ - and/or $\beta$-oxidation in patients with peroxisomal disorders causes markedly increased levels of branched chain fatty acids resulting in pathologies primarily in the peripheral nervous system and in cerebellum [16]. Pathological 
concentrations of phytanic acid were shown to depolarize mitochondria in different experimental conditions supposedly mediated by inhibition of complex I [50,51] or by a protonophoric mechanism of action [52]. However, we could rule out that the profound impairment of complex I activity in L-Pex5 knockout liver was due to the mildly increased levels of phytanic and pristanic acid. Indeed, even 1050 fold higher hepatic levels of these branched chain fatty acids, induced by feeding wild type mice a phytol enriched diet, did not affect complex I activity, although it induced morphological hepatic changes as described $[23,53]$.

Omega-oxidation of fatty acids in the ER, accounting for $5-10 \%$ of total fatty acid metabolism under normal conditions [38], converts (very) long chain fatty acids to DCAs and is catalyzed by cytochrome P450 enzymes. Formed DCAs depend strictly on peroxisomal $\beta$-oxidation for their catabolism to shorter chain lengths and after further degradation in mitochondria are finally excreted in urine [54-56]. Peroxisomal acyl-CoA oxidase (ACOX1) is one of the central enzymes involved in the $\beta$-oxidation of DCAs. Aco $\times 1^{-1}$ mice have been shown to develop steatohepatitis which was attributed to the accumulation of DCAs $[40,57]$. These di-anionic fatty acids can damage mitochondria and inhibit mitochondrial $\beta$-oxidation by dissipation of the mitochondrial proton gradient and uncoupling oxidative phosphorylation along with increased ROS generation during the oxidation process itself [38-40]. Liver mitochondria treated with Reye's Syndrome serum, which has as high as $54 \%$ of DCAs in total serum fatty acids, significantly uncoupled mitochondrial respiration and distorted mitochondrial ultrastructure [58]. Contrary to our expectations, we did not find increased levels of DCAs in L-Pex5 knockout livers, not even when mice were fed a DCA generating coconut diet (data not shown). This is surprising given that such diet strongly raised DCA levels in Mfp1 knockout mouse liver. Considering that in the latter mouse livers, despite extensive pathology that led to premature death [24], no changes at the level of complex I activity occur, we can exclude that DCA are at the origin of mitochondrial damage in L-Pex5- mice.

Bile acids are primarily synthesized in liver from cholesterol and peroxisomes are essential both for the side chain shortening by $\beta$-oxidation and the conjugation steps $[59,60]$. Inactivity of the $\beta$-oxidation leads to accumulation of C27 bile acid intermediates DHCA and THCA. In cholestatic liver disease characterized by accumulation of mature bile acids, mitochondrial abnormalities have been reported in the liver. For this reason the toxicity of bile acids has been extensively studied using either intact hepatic 
cells or isolated liver mitochondria of rodent or human origin. Bile acids have been shown to inhibit respiratory complexes, respiratory uncoupling, increased superoxide/ROS production, mitochondrial transition pore opening, apoptotic and necrotic cell death which was more prominent when hydrophobic bile acids were used [61-66]. The toxicity of the C27 bile acid intermediates in their conjugated or nonconjugated form was investigated by Ferdinandusse and colleagues using the rat hepatoma cell line McA-RH7777 [41]. The unconjugated C27 bile acids, in particular DHCA, were the most potent in inducing cell death and in inhibiting oxidative phosphorylation thereby increasing ROS production. Although unconjugated DHCA and THCA accumulate in L-Pex5 -liver, our data do not provide evidence that these destroy the inner mitochondrial membrane. Indeed, the levels of these bile acid species in liver of $\mathrm{Mfp}^{-/}$and $\mathrm{L}-\mathrm{Pex} 5^{-/}$mice at different ages did not correlate with crippled mitochondria in Pex5 deficiency from the neonatal period throughout adulthood, and preserved mitochondria in Mfp2 mutants at all ages.

In summary, we show that mitochondria in hepatocytes are more vulnerable to cell autonomous functional peroxisomal deficits than mitochondria in other cell types. As several peroxisome related metabolite abnormalities that are most prominent in peroxisome-deficient hepatocytes could not be linked to the mitochondrial defects, the molecular link between defective peroxisomes and inner mitochondrial membrane disruption remains mysterious. However, it needs to be emphasized that in this study overall levels of these metabolites were measured and further refinements are required to exclude changes in intermediates, e.g. carnitine-esters and CoA-esters. On the other hand, it cannot be excluded that a combination of deregulated metabolites causes the mitochondrial deficits. Alternatively, it needs to be envisaged that other than metabolic factors are involved.

\section{Acknowledgements:}

The authors thank Benny Das, Lies Pauwels, Evelyn De Schryver, Stanny Asselberghs for excellent technical assistance and Prof. Bernard Thorens (Lausanne, Switzerland) for providing liver samples of coconut diet fed Mfp1 knockout mice. This work was funded by grants from Fonds Wetenschappelijk Onderzoek Vlaanderen (G.0760.09) and KULeuven OT (08/40). ABS received a DBOF fellowship from KU Leuven (DBOF/10/059 to PPVV and MB). 


\section{FIGURE LEGENDS}

\section{Figure 1: Mitochondrial abnormalities are tissue selective}

(a,c,e) Complex I activities in tissues of conditional Pex5 knockout compared to the respective control (Ct) mice. Complex I activity is strongly reduced in adult (left panel) and P14 (right panel) livers of L-

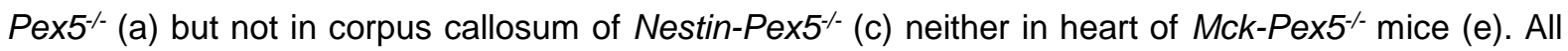
values are expressed as mean \pm SEM $(n=4-5) . P<0.01:{ }^{* *} ; p<0.001:{ }^{* \star *} L-P e x 5^{-/}$versus control mice. Electron micrographs of hepatocytes of P14 control and L-Pex5 $5^{-/}$mice (b) show severely altered mitochondrial ultrastructure while those of corpus callosum of Nestin-Pex5 $5^{-/}$mice (d) as well as heart of Mck-Pex5 $5^{-/}$mice (f) show normal mitochondrial ultrastructure.

Figure 2: DHA levels do not correlate with mitochondrial complex I activity

(a) DHA is depleted in the mitochondria of $L-P e x 5^{-\sim}$ liver and restored above levels in control mice after oral DHA supplementation. (b) The reduced complex I activity in the livers of $L-P e x 5^{-/}$mice is not altered after replenishing DHA levels. All values are expressed as mean \pm SEM $(n=3) . p<0.01:{ }^{* *}, p<0.001:{ }^{* * *}$ versus control untreated; $\mathrm{p}<0.001$ : \#\#\# L-Pex5\% mice treated with $\mathrm{DHA}$ versus $L-P e \times 5 \%$ mice. Plipid=phospholipid

\section{Figure 3: Branched chain fatty acid accumulation does not impair complex I activity}

(a) Complex I activity in the livers of wild type (WT) mice fed with phytol diet was not different from wild type mice on regular chow, despite significant accumulation of phytanic and pristanic acid. All values are expressed as mean \pm SEM $(n=3)$.

\section{Figure 4: DCA levels do not correlate with mitochondrial alterations}

(a) Relative levels of C3 to C14 DCAs in the livers of $L-P e \times 5^{--}$mice on normal chow (left panel). The sum of all DCAs measured in control liver was set to $100 \%$ and the value for each DCA in control and L-Pex5 $\%$ mice was calculated as $\%$ of this sum, taking into account tissue weight and the internal standard methyl malonic acid. Total DCA level in L-Pex5 $\%$ liver was $80 \%$ of total DCA level in control liver (right panel). (b) Complex I activity in the livers of $M f p 1 \%$ mice was not different from controls, both fed with coconut diet. All values are expressed as mean \pm SEM $(n=4) . p<0.001{ }^{* * *} L-P e x 5^{-}$versus control mice.

Figure 5: Accumulating immature C27 bile acids are not associated with complex I impairment 
(a) The ratio of immature $\mathrm{C} 27$ to mature $\mathrm{C} 24$ bile acids is significantly increased in the livers of adult $L$ $P e x 5^{-/}$and even more in $\mathrm{Mfp}^{-}$mice in comparison to littermate controls. (b) Complex I activity in the livers of $\mathrm{Mfp2}^{--}$mice is not impaired in comparison to littermate controls. (c-d) Absolute values of

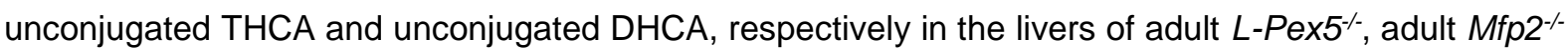
, E18.5 Pex5 and E18.5 Mfp2 $\%$ mice. Unconjugated DHCA is the sum of unconjugated forms of DHCA and $\triangle 24-\mathrm{DHCA}$ while unconjugated THCA is the sum of unconjugated forms of THCA, $\triangle 24-\mathrm{THCA}, \mathrm{OH}-$ THCA and $\triangle 24-\mathrm{OH}-\mathrm{THCA}$. Observed profiles of unconjugated forms of THCA and DHCA in these mice do not correlate with the mitochondrial anomalies. All values are expressed as mean $\pm \operatorname{SEM}(n=4), L$ Pex5 ${ }^{-\sim}$, Pex5 ${ }^{-/}$and $\mathrm{Mfp}^{-/}$mice versus respective control mice $\mathrm{p}<0.05:{ }^{*} ; \mathrm{p}<0.01:^{*} ; \mathrm{p}<0.001$ : $^{* * *}$.

\section{Supplementary Figure 1: Mitochondrial abnormalities are tissue selective}

Complex I activities in brain cortex (a) and spinal cord (b) of Nestin-Pex5\% mice along with their respective littermate controls $(\mathrm{Ct})$. No significant differences were found in both tissues when compared to their respective controls. (c) Electron micrographs of brain cortex of control and Nestin-Pex $5^{-/}$mice showing normal mitochondrial ultra-structure.

\section{Supplementary Figure 2: Inactivation of the Pex5 gene in Mck-Pex5 knockout mice}

(a) Northern blot analysis shows a truncated Pex5 transcript in heart and skeletal muscle of Mck-Pex5- mice as compared to controls (left panel), but not in liver, kidney and spleen (right panel) indicating selective recombination of the Pex5 gene in the targeted tissue. Reduced levels of plasmalogens in heart (b) and skeletal muscle (c) of $M c k-P e x 5^{-/}$mice demonstrate effective inactivation of peroxisomal function in these tissues. 


\section{References}

1. Goldfischer, S., et al., Peroxisomal and mitochondrial defects in the cerebro-hepato-renal syndrome. Science, 1973. 182(4107): p. 62-4.

2. Mooi, W.J., et al., Ultrastructure of the liver in th cerebrohepatorenal syndrome of Zellweger. Ultrastruct Pathol, 1983. 5(2-3): p. 135-44.

3. Mathis, R.K., et al., Liver in the cerebro-hepato-renal syndrome: defective bile acid synthesis and abnormal mitochondria. Gastroenterology, 1980. 79(6): p. 1311-7.

4. Aubourg, P., et al., The cerebro-hepato-renal (Zellweger) syndrome: lamellar lipid profiles in adrenocortical, hepatic mesenchymal, astrocyte cells and increased levels of very long chain fatty acids and phytanic acid in the plasma. J Neurol Sci, 1985. 69(1-2): p. 9-25.

5. Sarnat, H.B., et al., Mitochondrial myopathy of cerebro-hepato-renal (Zellweger) syndrome. Can J Neurol Sci, 1983. 10(3): p. 170-7.

6. Salpietro, V., et al., Zellweger syndrome and secondary mitochondrial myopathy. Eur J Pediatr, 2014.

7. Muller-Hocker, J., et al., Mitochondrial myopathy with loosely coupled oxidative phosphorylation in a case of Zellweger syndrome. A cytochemical-ultrastructural study. Virchows Arch B Cell Pathol Incl Mol Pathol, 1984. 45(2): p. 125-38.

8. Trijbels, J.M., et al., Biochemical studies in the liver and muscle of patients with Zellweger syndrome. Pediatr Res, 1983. 17(6): p. 514-7.

9. Baes, M., et al., A mouse model for Zellweger syndrome. Nat Genet, 1997. 17(1): p. 49-57.

10. Keane, M.H., et al., Bile acid treatment alters hepatic disease and bile acid transport in peroxisome-deficient PEX2 Zellweger mice. Hepatology, 2007. 45(4): p. 982-97.

11. Baumgart, E., et al., Mitochondrial alterations caused by defective peroxisomal biogenesis in a mouse model for Zellweger syndrome (PEX5 knockout mouse). Am J Pathol, 2001. 159(4): p. 1477-94.

12. Dirkx, R., et al., Absence of peroxisomes in mouse hepatocytes causes mitochondrial and $E R$ abnormalities. Hepatology, 2005. 41(4): p. 868-78.

13. Peeters, A., et al., Carbohydrate metabolism is perturbed in peroxisome-deficient hepatocytes due to mitochondrial dysfunction, AMP-activated protein kinase (AMPK) activation, and peroxisome proliferator-activated receptor gamma coactivator 1alpha (PGC1alpha) suppression. J Biol Chem, 2011. 286(49): p. 42162-79.

14. Peeters, A., et al., Mitochondria in peroxisome-deficient hepatocytes exhibit impaired respiration, depleted DNA, and PGC-1alpha independent proliferation. Biochim Biophys Acta, 2015. 1853(2): p. 285-98.

15. Al-Hussaini, A., et al., Clinical and molecular characteristics of mitochondrial DNA depletion syndrome associated with neonatal cholestasis and liver failure. J Pediatr, 2014. 164(3): p. 553-9 e1-2.

16. Van Veldhoven, P.P., Biochemistry and genetics of inherited disorders of peroxisomal fatty acid metabolism. J Lipid Res, 2010. 51(10): p. 2863-95.

17. $\mathrm{Qi}, \mathrm{C}$., et al., Absence of spontaneous peroxisome proliferation in enoyl-CoA Hydratase/L-3hydroxyacyl-CoA dehydrogenase-deficient mouse liver. Further support for the role of fatty acyl CoA oxidase in PPARalpha ligand metabolism. J Biol Chem, 1999. 274(22): p. 15775-80.

18. Baes, M., et al., Inactivation of the peroxisomal multifunctional protein-2 in mice impedes the degradation of not only 2-methyl-branched fatty acids and bile acid intermediates but also of very long chain fatty acids. J Biol Chem, 2000. 275(21): p. 16329-36.

19. Baes, M., et al., Generation of Pex5-loxP mice allowing the conditional elimination of peroxisomes. Genesis, 2002. 32(2): p. 177-8.

20. Hulshagen, L., et al., Absence of functional peroxisomes from mouse CNS causes dysmyelination and axon degeneration. J Neurosci, 2008. 28(15): p. 4015-27. 
21. Bruning, J.C., et al., A muscle-specific insulin receptor knockout exhibits features of the metabolic syndrome of NIDDM without altering glucose tolerance. Mol Cell, 1998. 2(5): $p$. 559-69.

22. Lalancette-Hebert, M., et al., Accumulation of dietary docosahexaenoic acid in the brain attenuates acute immune response and development of postischemic neuronal damage. Stroke, 2011. 42(10): p. 2903-9.

23. Mezzar, S., et al., Phytol-induced pathology in 2-hydroxyacyl-CoA lyase (HACL1) deficient mice. Evidence for a second non-HACL1-related lyase. Biochim Biophys Acta, 2017. 1862(9): p. $972-990$.

24. Ding, J., et al., The peroxisomal enzyme L-PBE is required to prevent the dietary toxicity of medium-chain fatty acids. Cell Rep, 2013. 5(1): p. 248-58.

25. Ferdinandusse, S., et al., Developmental changes of bile acid composition and conjugation in L- and D-bifunctional protein single and double knockout mice. J Biol Chem, 2005. 280(19): p. 18658-66.

26. Janssen, A., et al., Docosahexaenoic acid deficit is not a major pathogenic factor in peroxisome-deficient mice. Lab Invest, 2000. 80(1): p. 31-5.

27. Baes, M., et al., The neuronal migration defect in mice with Zellweger syndrome (Pex5 knockout) is not caused by the inactivity of peroxisomal beta-oxidation. J Neuropathol Exp Neurol, 2002. 61(4): p. 368-74.

28. Van Veldhoven, P.P. and G.P. Mannaerts, Inorganic and organic phosphate measurements in the nanomolar range. Anal Biochem, 1987. 161(1): p. 45-8.

29. Roels, F., M. Espeel, and D. De Craemer, Liver pathology and immunocytochemistry in congenital peroxisomal diseases: a review. J Inherit Metab Dis, 1991. 14(6): p. 853-75.

30. da Silva, T.F., et al., Peripheral nervous system plasmalogens regulate Schwann cell differentiation and myelination. J Clin Invest, 2014. 124(6): p. 2560-70.

31. Bottelbergs, A., et al., Peroxisome deficiency but not the defect in ether lipid synthesis causes activation of the innate immune system and axonal loss in the central nervous system. J Neuroinflammation, 2012. 9: p. 61.

32. Ferdinandusse, S., et al., Studies on the metabolic fate of $n-3$ polyunsaturated fatty acids. J Lipid Res, 2003. 44(10): p. 1992-7.

33. Rapoport, S.I., M. Igarashi, and F. Gao, Quantitative contributions of diet and liver synthesis to docosahexaenoic acid homeostasis. Prostaglandins Leukot Essent Fatty Acids, 2010. 82(46): p. 273-6.

34. Ronicke, S., et al., The influence of the branched-chain fatty acids pristanic acid and Refsum disease-associated phytanic acid on mitochondrial functions and calcium regulation of hippocampal neurons, astrocytes, and oligodendrocytes. Neurobiol Dis, 2009. 36(2): p. 40110.

35. Okita, R.T. and J.R. Okita, Cytochrome P450 4A fatty acid omega hydroxylases. Curr Drug Metab, 2001. 2(3): p. 265-81.

36. Johnson, E.F., et al., Regulation of P450 $4 A$ expression by peroxisome proliferator activated receptors. Toxicology, 2002. 181-182: p. 203-6.

37. Peeters, A., et al., Hepatosteatosis in peroxisome deficient liver despite increased betaoxidation capacity and impaired lipogenesis. Biochimie, 2011. 93(10): p. 1828-38.

38. Hardwick, J.P., Cytochrome P450 omega hydroxylase (CYP4) function in fatty acid metabolism and metabolic diseases. Biochem Pharmacol, 2008. 75(12): p. 2263-75.

39. Passi, S., et al., Antimitochondrial effect of saturated medium chain length (C8-C13) dicarboxylic acids. Biochem Pharmacol, 1984. 33(1): p. 103-8.

40. Reddy, J.K. and M.S. Rao, Lipid metabolism and liver inflammation. II. Fatty liver disease and fatty acid oxidation. Am J Physiol Gastrointest Liver Physiol, 2006. 290(5): p. G852-8.

41. Ferdinandusse, S., et al., Toxicity of peroxisomal C27-bile acid intermediates. Mol Genet Metab, 2009. 96(3): p. 121-8. 
42. Jia, Y., et al., Overexpression of peroxisome proliferator-activated receptor-alpha (PPARalpha)-regulated genes in liver in the absence of peroxisome proliferation in mice deficient in both L- and D-forms of enoyl-CoA hydratase/dehydrogenase enzymes of peroxisomal beta-oxidation system. J Biol Chem, 2003. 278(47): p. 47232-9.

43. Cui, J.Y., et al., Bile acids via FXR initiate the expression of major transporters involved in the enterohepatic circulation of bile acids in newborn mice. Am J Physiol Gastrointest Liver Physiol, 2012. 302(9): p. G979-96.

44. Baarine, M., et al., $A B C D 1$ deletion-induced mitochondrial dysfunction is corrected by SAHA: implication for adrenoleukodystrophy. J Neurochem, 2015. 133(3): p. 380-96.

45. Lopez-Erauskin, J., et al., Impaired mitochondrial oxidative phosphorylation in the peroxisomal disease X-linked adrenoleukodystrophy. Hum Mol Genet, 2013. 22(16): p. 3296305.

46. Igarashi, M., et al., Docosahexaenoic acid synthesis from alpha-linolenic acid by rat brain is unaffected by dietary n-3 PUFA deprivation. J Lipid Res, 2007. 48(5): p. 1150-8.

47. Khairallah, R.J., et al., Improved mitochondrial function with diet-induced increase in either docosahexaenoic acid or arachidonic acid in membrane phospholipids. PLoS One, 2012. 7(3): p. e34402.

48. Stanley, W.C., R.J. Khairallah, and E.R. Dabkowski, Update on lipids and mitochondrial function: impact of dietary n-3 polyunsaturated fatty acids. Curr Opin Clin Nutr Metab Care, 2012. 15(2): p. 122-6.

49. Eckert, G.P., U. Lipka, and W.E. Muller, Omega-3 fatty acids in neurodegenerative diseases: focus on mitochondria. Prostaglandins Leukot Essent Fatty Acids, 2013. 88(1): p. 105-14.

50. Schonfeld, P. and G. Reiser, Rotenone-like action of the branched-chain phytanic acid induces oxidative stress in mitochondria. J Biol Chem, 2006. 281(11): p. 7136-42.

51. Reiser, G., P. Schonfeld, and S. Kahlert, Mechanism of toxicity of the branched-chain fatty acid phytanic acid, a marker of Refsum disease, in astrocytes involves mitochondrial impairment. Int J Dev Neurosci, 2006. 24(2-3): p. 113-22.

52. Komen, J.C., et al., Phytanic acid impairs mitochondrial respiration through protonophoric action. Cell Mol Life Sci, 2007. 64(24): p. 3271-81.

53. Mackie, J.T., et al., Phytol-induced hepatotoxicity in mice. Toxicol Pathol, 2009. 37(2): p. 2018.

54. Sanders, R.J., et al., Omega-oxidation of very long-chain fatty acids in human liver microsomes. Implications for X-linked adrenoleukodystrophy. J Biol Chem, 2006. 281(19): p. 13180-7.

55. Sanders, R.J., et al., Characterization of the human omega-oxidation pathway for omegahydroxy-very-long-chain fatty acids. FASEB J, 2008. 22(6): p. 2064-71.

56. Nguyen, S.D., M. Baes, and P.P. Van Veldhoven, Degradation of very long chain dicarboxylic polyunsaturated fatty acids in mouse hepatocytes, a peroxisomal process. Biochim Biophys Acta, 2008. 1781(8): p. 400-5.

57. Reddy, J.K. and T. Hashimoto, Peroxisomal beta-oxidation and peroxisome proliferatoractivated receptor alpha: an adaptive metabolic system. Annu Rev Nutr, 2001. 21: p. 193230.

58. Tonsgard, J.H. and G.S. Getz, Effect of Reye's syndrome serum on isolated chinchilla liver mitochondria. J Clin Invest, 1985. 76(2): p. 816-25.

59. Wanders, R.J. and S. Ferdinandusse, Peroxisomes, peroxisomal diseases, and the hepatotoxicity induced by peroxisomal metabolites. Curr Drug Metab, 2012. 13(10): p. 140111.

60. Pellicoro, A., et al., Human and rat bile acid-CoA:amino acid $\mathrm{N}$-acyltransferase are liverspecific peroxisomal enzymes: implications for intracellular bile salt transport. Hepatology, 2007. 45(2): p. 340-8. 
61. Benz, C., et al., Effect of tauroursodeoxycholic acid on bile acid-induced apoptosis in primary human hepatocytes. Eur J Clin Invest, 2000. 30(3): p. 203-9.

62. Yerushalmi, B., et al., Bile acid-induced rat hepatocyte apoptosis is inhibited by antioxidants and blockers of the mitochondrial permeability transition. Hepatology, 2001. 33(3): p. 61626.

63. Sokol, R.J., et al., Human hepatic mitochondria generate reactive oxygen species and undergo the permeability transition in response to hydrophobic bile acids. J Pediatr Gastroenterol Nutr, 2005. 41(2): p. 235-43.

64. Rolo, A.P., C.M. Palmeira, and K.B. Wallace, Mitochondrially mediated synergistic cell killing by bile acids. Biochim Biophys Acta, 2003. 1637(1): p. 127-32.

65. Palmeira, C.M. and A.P. Rolo, Mitochondrially-mediated toxicity of bile acids. Toxicology, 2004. 203(1-3): p. 1-15.

66. Sokol, R.J., et al., Role of oxidant stress in the permeability transition induced in rat hepatic mitochondria by hydrophobic bile acids. Pediatr Res, 2001. 49(4): p. 519-31. 


\section{Figure 1}

a

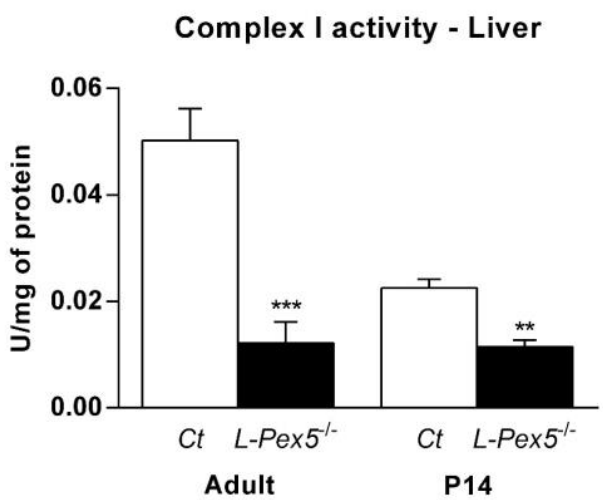

b
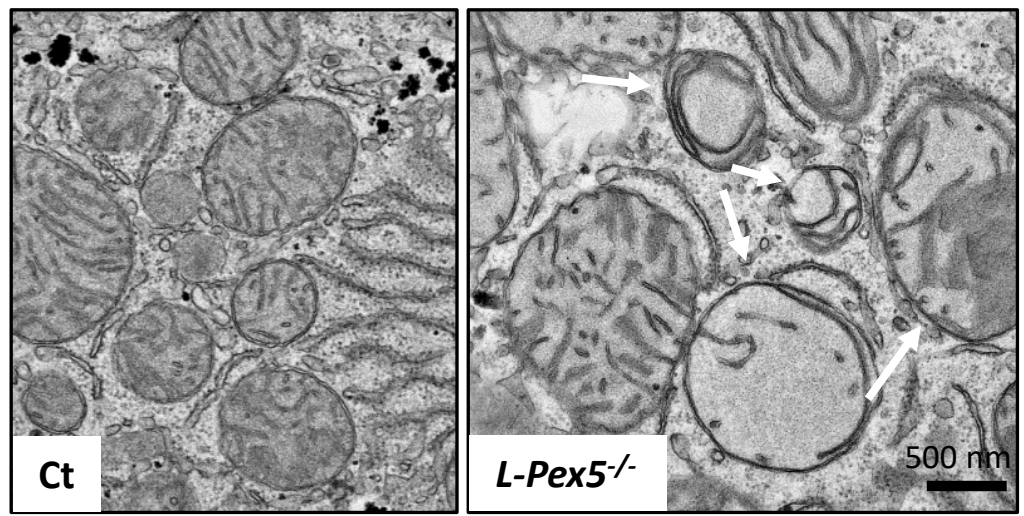

C

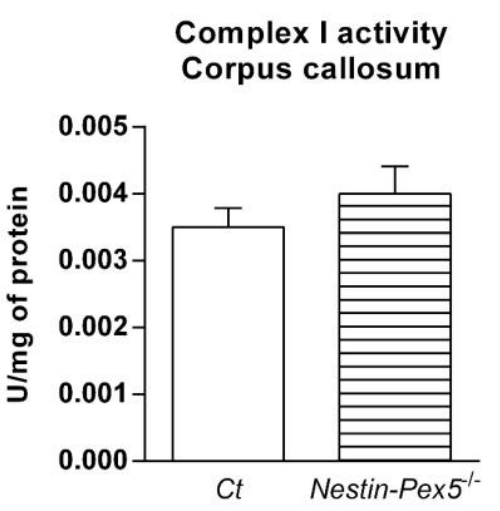

d
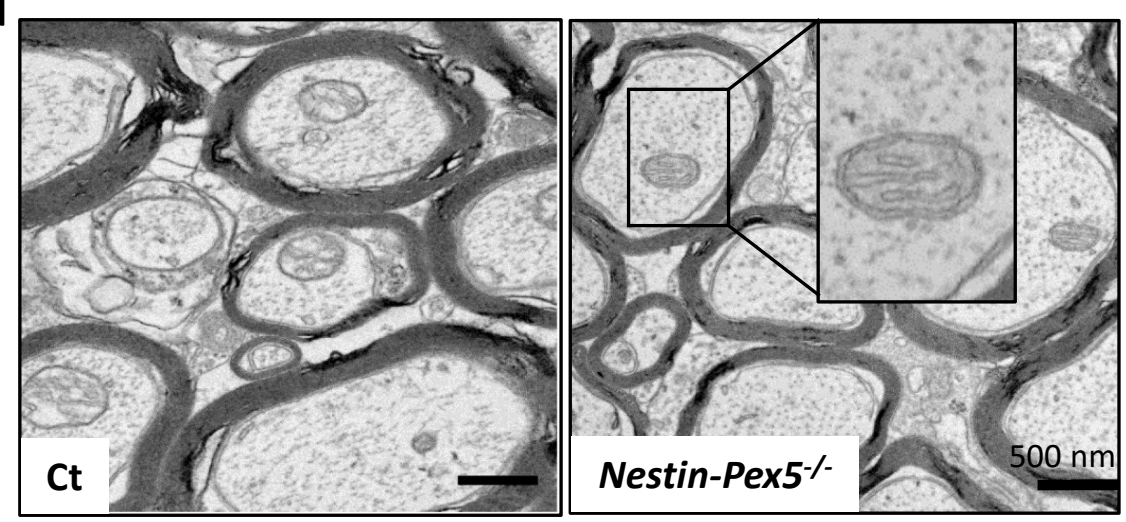

e
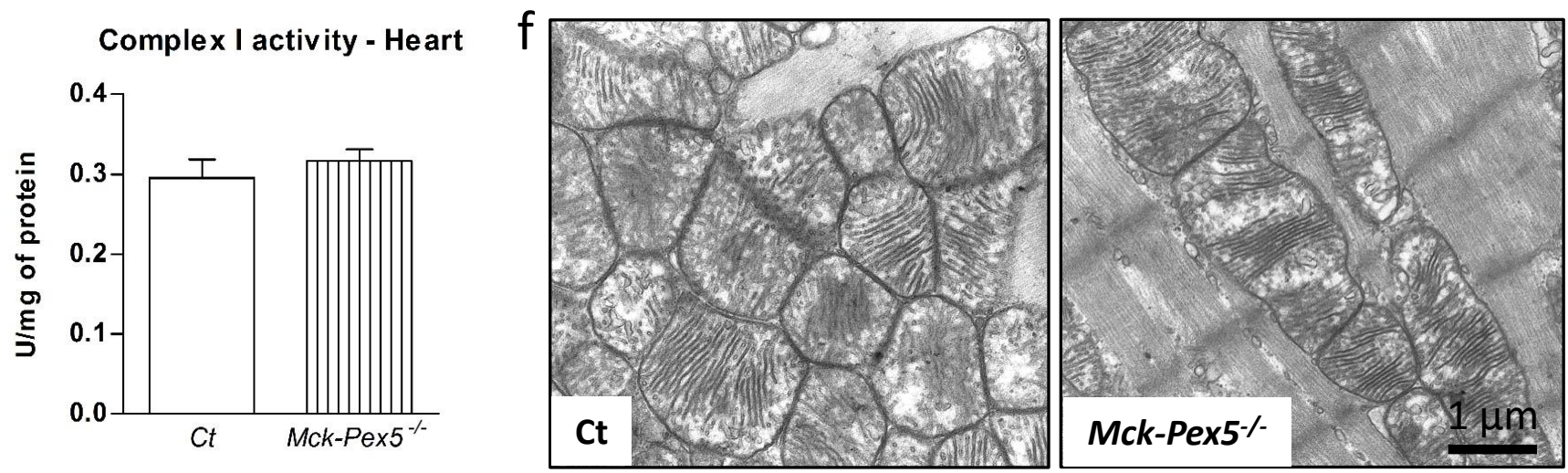
Figure 2

a

DHA - Liver mitochondria

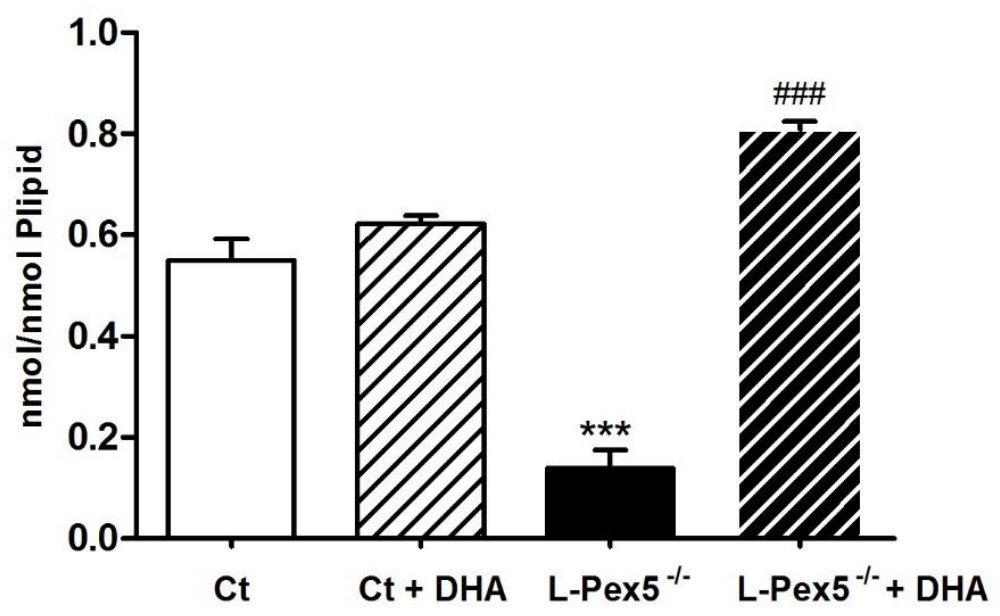

b

Complex I activity - Liver

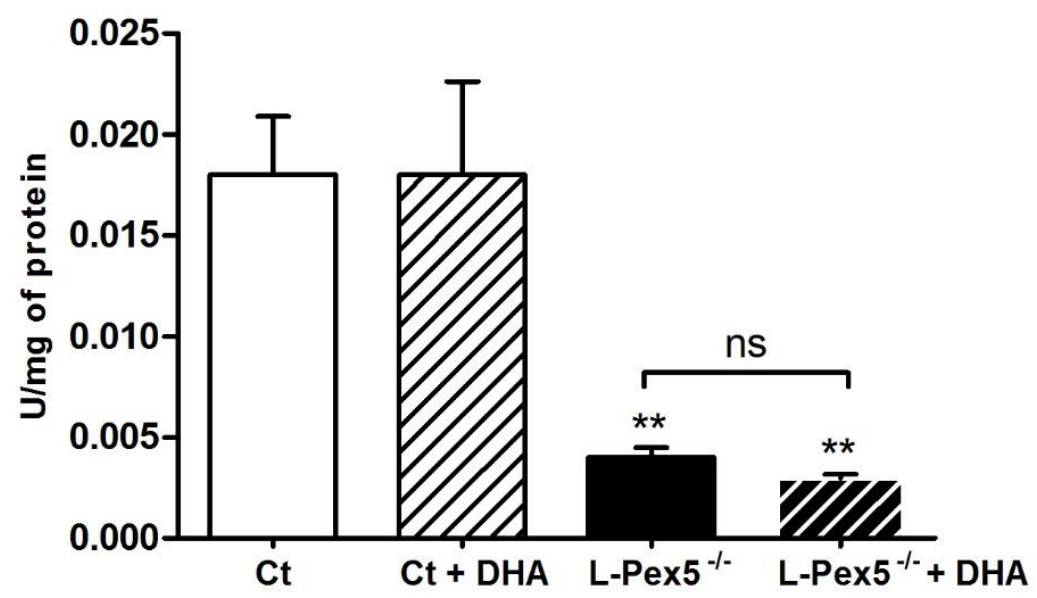


Figure 3

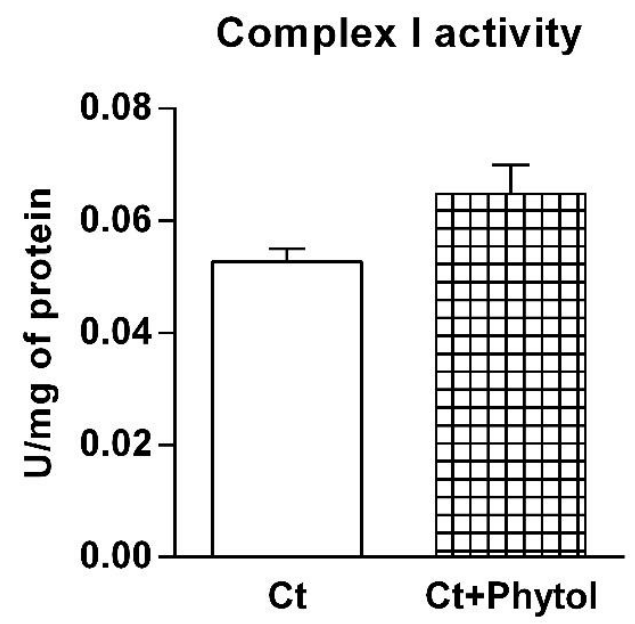


Figure 4

a

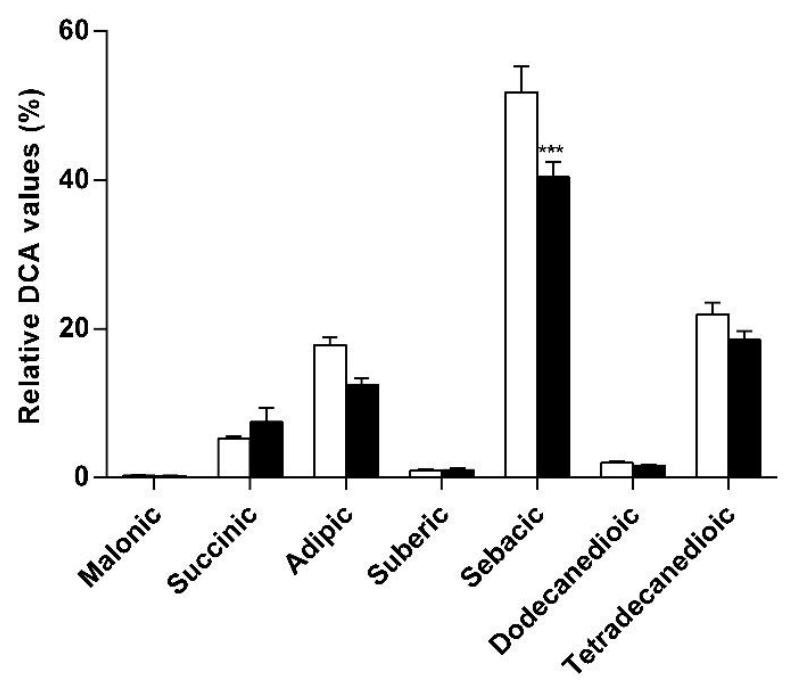

b

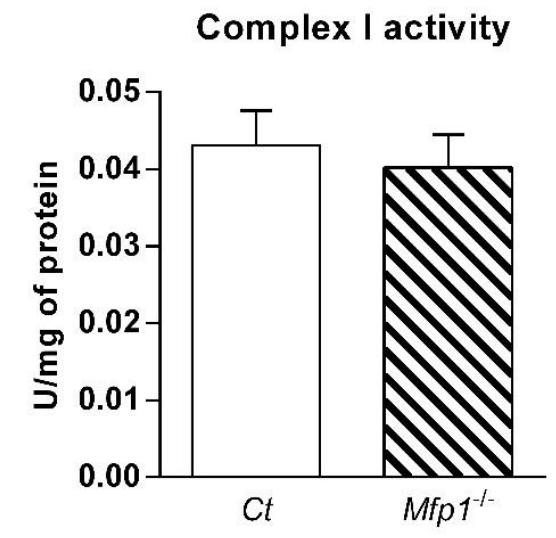

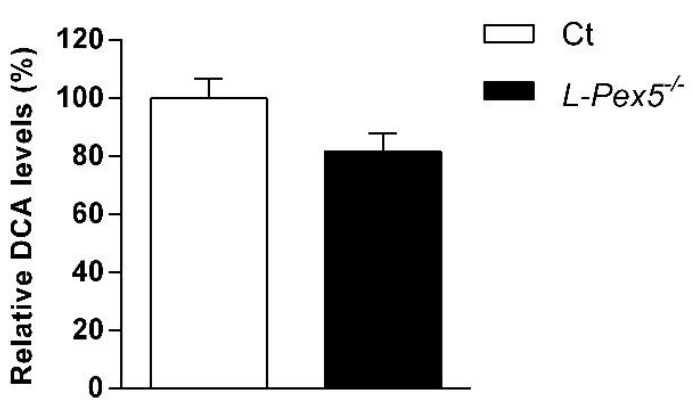


Figure 5

a

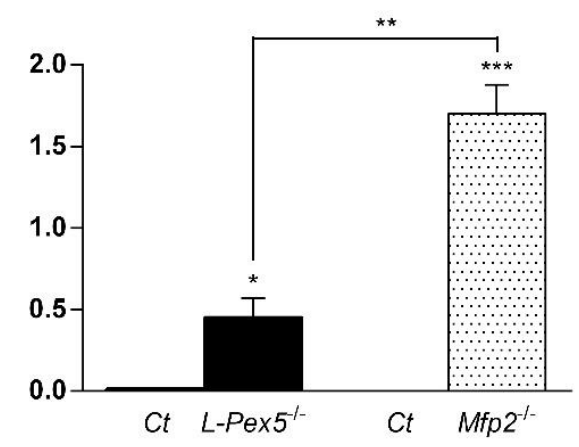

C

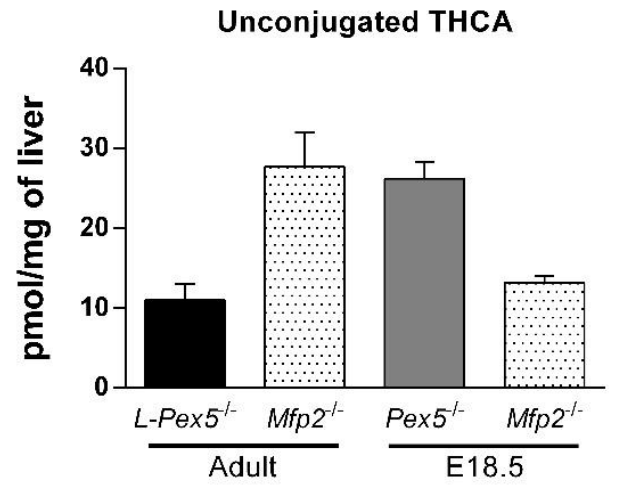

b Complex I activity

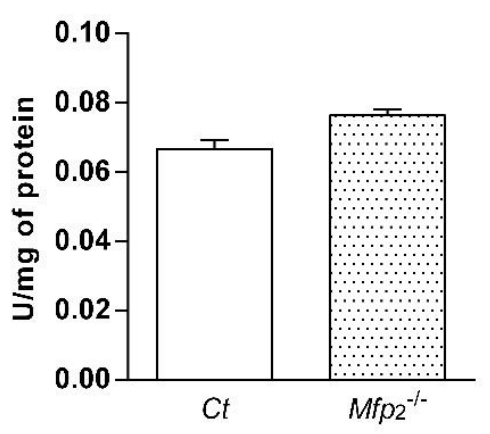

d

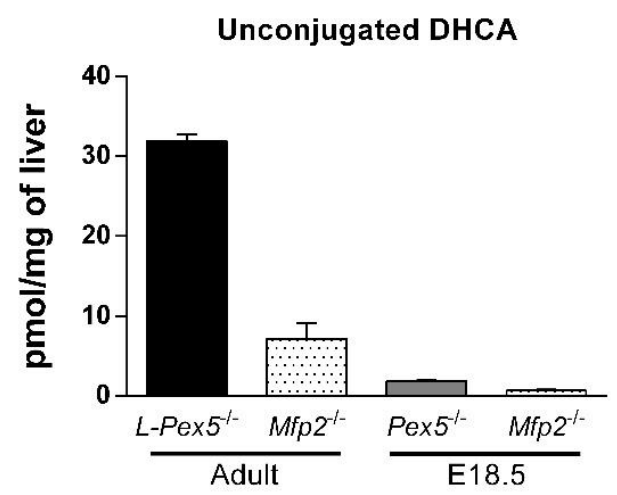




\section{Supplementary figure 1}

a

Complex I activity - Brain Cortex

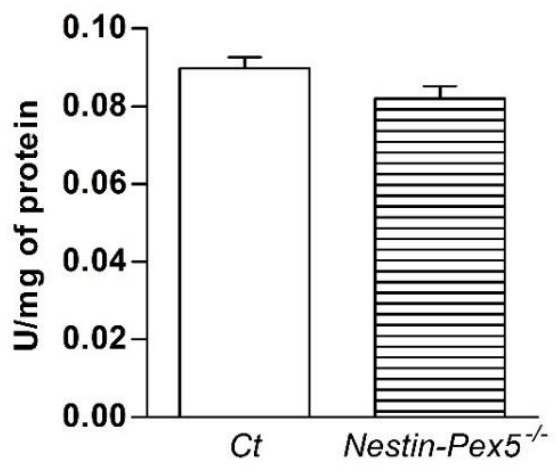

C

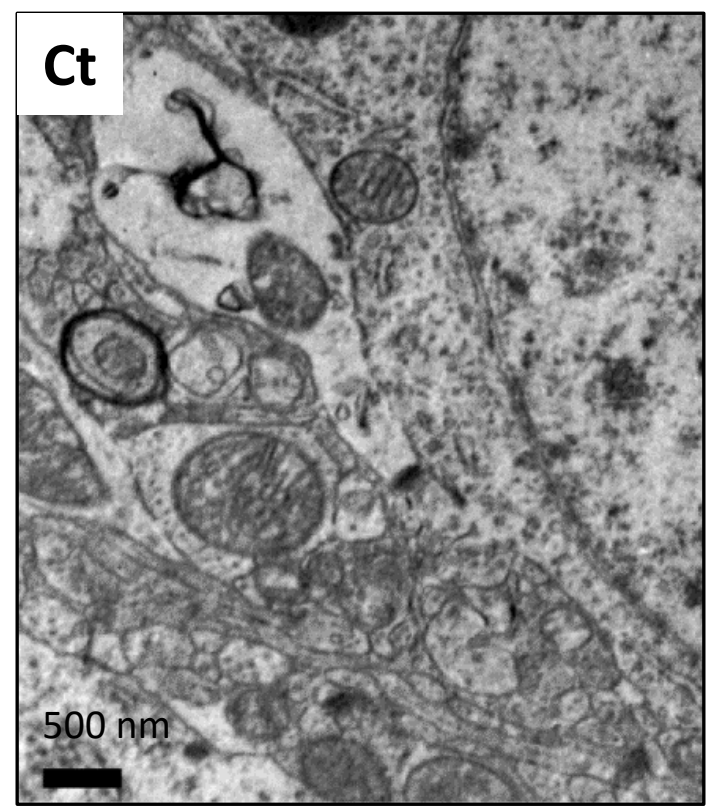

b Complex I activity - Spinal cord

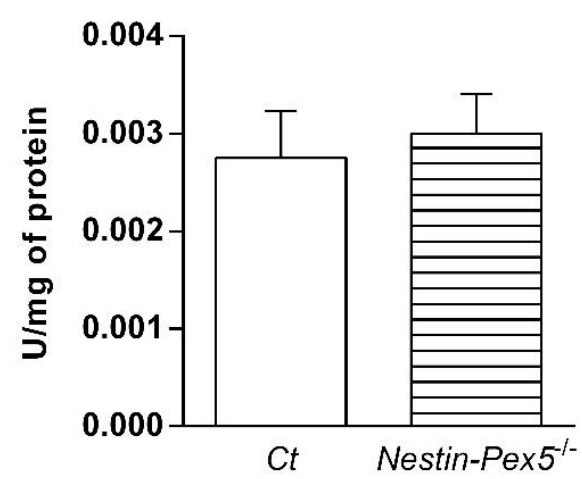




\section{Supplementary figure 2}

a
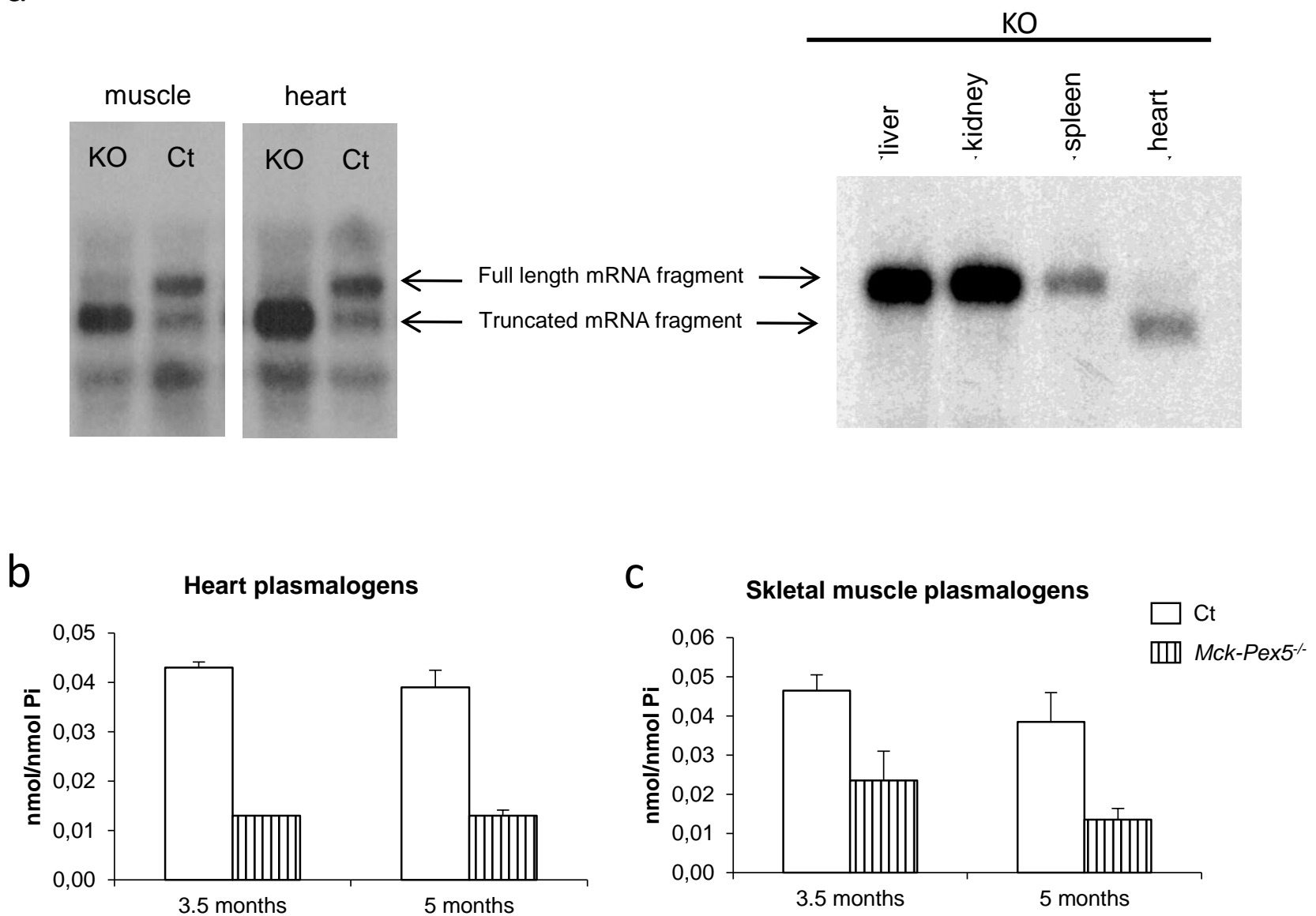
Table 1: Conjugated and unconjugated $\mathrm{C} 24$ and $\mathrm{C} 27$ bile acids in liver of $\mathrm{Ct}, \mathrm{L}-\mathrm{Pex} 5^{\%}, \mathrm{Pex} 5^{\%}$ and $\mathrm{Mfp2} \%$ mice.

\begin{tabular}{|c|c|c|c|c|c|c|c|c|}
\hline Age & Genotype & Total & $\begin{array}{l}\text { Conjugated } \\
\text { C24 }\end{array}$ & $\begin{array}{l}\text { Unconjugated } \\
\text { C24 }\end{array}$ & $\begin{array}{l}\text { Conjugated } \\
\text { C27 }\end{array}$ & $\begin{array}{l}\text { Unconjugated } \\
\text { C27 }\end{array}$ & $\begin{array}{l}\text { Unconjugated } \\
\text { DHCA }\end{array}$ & $\begin{array}{l}\text { Unconjugated } \\
\text { THCA }\end{array}$ \\
\hline & $\mathrm{Ct}$ & $319 \pm 75$ & $281 \pm 62$ & $36 \pm 13$ & $0.35 \pm 0.30$ & $1.66 \pm 0.41$ & $0.53 \pm 0.19$ & $1.12 \pm 0.61$ \\
\hline & $L-P e \times 5^{-}$ & $189 \pm 34$ & $142 \pm 44$ & $8.1 \pm 4.0$ & $7.0 \pm 0.5^{*}$ & $41 \pm 2 * * *$ & $31.92 \pm 0.77^{* * *}$ & $10.97 \pm 4.06^{* *}$ \\
\hline & $\mathrm{Ct}$ & $489 \pm 32$ & $457 \pm 36$ & $31 \pm 4$ & $0.21 \pm 0.10$ & $0.7 \pm 0.2$ & $0.04 \pm 0.04$ & $0.33 \pm 0.39$ \\
\hline & $M f p 2^{-/-}$ & $192 \pm 25^{* * *}$ & $72 \pm 15^{* * *}$ & $5.1 \pm 2.0^{* *}$ & $87 \pm 11^{* * *}$ & $36 \pm 8^{* *}$ & $7.14 \pm 1.95^{*}$ & $27.64 \pm 8.59 * *$ \\
\hline & $\mathrm{Ct}$ & $123 \pm 15$ & $121 \pm 15$ & $0.54 \pm 0.08$ & $0.27 \pm 0.05$ & $0.30 \pm 0.05$ & $0.15 \pm 0.04$ & $0.15 \pm 0.01$ \\
\hline & $P e \times 5^{-/-}$ & $39 \pm 3 * * *$ & $8.9 \pm 1.6^{* * *}$ & $1.16 \pm 0.07^{* * *}$ & $0.63 \pm 0.11^{*}$ & $28.06 \pm 2.13^{* * *}$ & $1.86 \pm 0.16^{* * *}$ & $26.19 \pm 2.12^{* * *}$ \\
\hline & $\mathrm{Ct}$ & $91 \pm 14$ & $90 \pm 14$ & $0.37 \pm 0.07$ & $0.49 \pm 0.11$ & $0.31 \pm 0.02$ & $0.09 \pm 0.01$ & $0.22 \pm 0.03$ \\
\hline & $M f p 2^{-/-}$ & $38 \pm 3^{* *}$ & $14.0 \pm 2.1^{* *}$ & $3.46 \pm 0.31 * * *$ & $6.39 \pm 0.67^{* * *}$ & $13.83 \pm 0.7^{* * *}$ & $0.66 \pm 0.1^{* *}$ & $13.17 \pm 0.76^{* * *}$ \\
\hline
\end{tabular}

All values are in pmol/mg liver. Values are expressed as mean \pm SEM. Unconjugated DHCA is the sum of unconjugated DHCA and $\triangle 24-\mathrm{DHCA}$

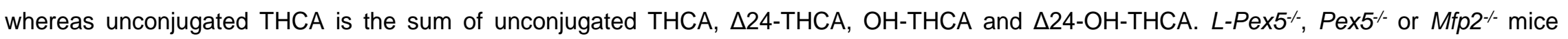
versus respective control mice; $p<0.05:{ }^{*} ; p<0.01:{ }^{* *} ; p<0.001:{ }^{* * *}$. 